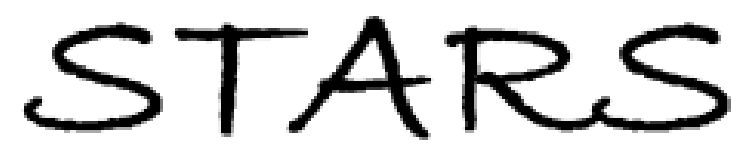

University of Central Florida

STARS

Faculty Bibliography 2000s

Faculty Bibliography

$1-1-2009$

\title{
Novel method for the fabrication of spatially variant structures
}

Pradeep Srinivasan

University of Central Florida

Zachary A. Roth

Menelaos K. Poutous

Eric G. Johnson

Find similar works at: https://stars.library.ucf.edu/facultybib2000

University of Central Florida Libraries http://library.ucf.edu

This Article is brought to you for free and open access by the Faculty Bibliography at STARS. It has been accepted for inclusion in Faculty Bibliography 2000 s by an authorized administrator of STARS. For more information, please contactSTARS@ucf.edu.

\section{Recommended Citation}

Srinivasan, Pradeep; Roth, Zachary A.; Poutous, Menelaos K.; and Johnson, Eric G., "Novel method for the fabrication of spatially variant structures" (2009). Faculty Bibliography 2000s. 2175.

https://stars.library.ucf.edu/facultybib2000/2175

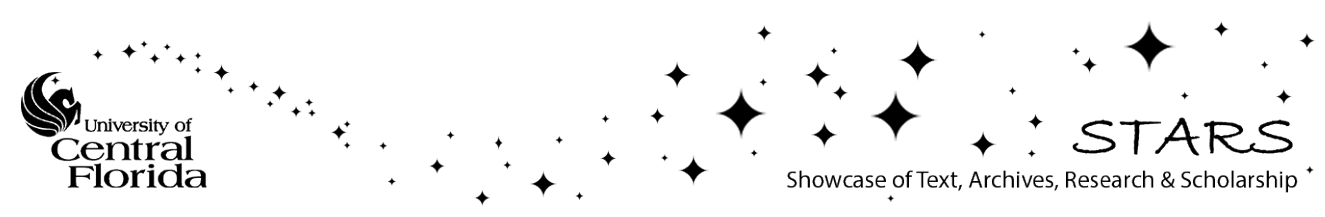




\section{Novel method for the fabrication of spatially variant structures}

\author{
Pradeep Srinivasan \\ University of Central Florida \\ College of Optics and Photonics/CREOL \\ 4000 Central Florida Boulevard \\ Orlando, Florida 32816
}

\author{
Zachary A. Roth \\ Menelaos K. Poutous \\ Eric G. Johnson \\ University of North Carolina \\ Center for Optoelectronics and Optical \\ Communications \\ 9201 University City Boulevard \\ Charlotte, North Carolina 28223 \\ E-mail: egjohnso@uncc.edu
}

\begin{abstract}
Spatially varying grating structures formed at the subwavelength scale behave as a layer with an artificial effective refractive index that is dependent on the local fill fraction. We describe a novel technique to pattern gratings with a spatially varying fill fraction using a simple two-step exposure process. The first exposure forms a partial latent image of a grating in the photoresist. The resist is then saturated by overlaying an exposure with an analog spatially varying intensity, generated by using a phase-only masking technique. The cumulative exposure dose from the two steps was designed so that the point of minimum intensity will still develop the photoresist through, in all the spaces in the grating. By varying the exposure window around the saturation dose, the fill fraction of the patterned gratings was modulated; thus, the size of the space cleared at any location in the grating is a scalable function of the local cumulative dose delivered. Constant feature height is achieved across the patterned area by keeping the second exposure dose below the resist threshold exposure value. The exposure process was modeled numerically to predict the relationship between the local dose and patterned fill fraction. This technique enables rapid, low-cost fabrication of apodized grating structures for applications in diffractive optics technology. () 2009 Society of Photo-Optical Instrumentation Engineers. [DOI: 10.1117/1.3066798]
\end{abstract}

Subject terms: diffractive optics; diffractive optical elements; apodization; space varying structures; micro-optics; lithography; gratings; optical fabrications.

Paper 08026R received Apr. 27, 2008; revised manuscript received Sep. 20, 2008; accepted for publication Nov. 21, 2008; published online Feb. 12, 2009. This paper is a revision of a paper presented at the SPIE Conference on Advanced Fabrication Technologies for Micro/Nano Optics and Photonics, January 2008, San Jose, California. The paper presented there appears (unrefereed) in SPIE Proceedings Vol. 6883.

\section{Introduction}

Space-variant periodic structures have been widely used in optical devices. They provide novel optical functionalities and improved performance. In the case of grating structures formed at the subwavelength scale, the effective refractive index of a grating layer is a function of the grating periodto-wavelength ratio and the fill fraction.

The effective refractive index dependence on the duty cycle of a periodic structure has been exploited in spatially varying elements to realize broadband response in diffractive optical devices, such as blazed grating structures. ${ }^{1,2}$ The phase retardation of a blazed grating is achieved by varying the effective refractive index across the element instead of modulating the thickness of the structure. By varying the fill fraction in a regime that provides the same differential phase retardation across the element, for a certain desired range of wavelengths, the blazed grating can be optimized to perform with very high efficiency over a broader wavelength band in comparison to classical echelette gratings. By locally controlling the retardation experienced by the orthogonal polarization states at various points in the incident beam, polarization converting elements have also been realized. ${ }^{3-5}$ Apodization or spatial

$1932-5150 / 2009 / \$ 25.00$ @ 2009 SPIE variation of the filling fraction of subwavelength gratingbased filters, such as distributed Bragg reflectors has been shown to improve the optical response by improving the side-lobe suppression ratio. ${ }^{6,7}$ Subwavelength structures with spatially varying duty cycles have also been employed for encoding phase functions in holograms for encryption applications.

Grating structures can be fabricated using a variety of techniques, including electron beam lithography, ultraviolet contact and projection lithography, and holographic techniques, depending on the desired grating specifications. Spatially variable duty cycle gratings have been formed mainly using electron beam lithography ${ }^{1,2}$ although e-beam lithography can achieve complex patterns, it is an inherently serial fabrication process. ${ }^{9}$ Hence, the patterning time is directly proportional to the desired fidelity of the structures and the total size of the device area. Uniform dutycycle variation is hard to implement by use of proximityeffect methods, such as e-beam lithography, to vary the grating feature widths. ${ }^{10}$ This makes practical implementation of optical elements of this type prohibitively expensive.

\section{Our Approach}

In this paper, we describe a novel method to fabricate space-variant grating structures using a simple two-step 


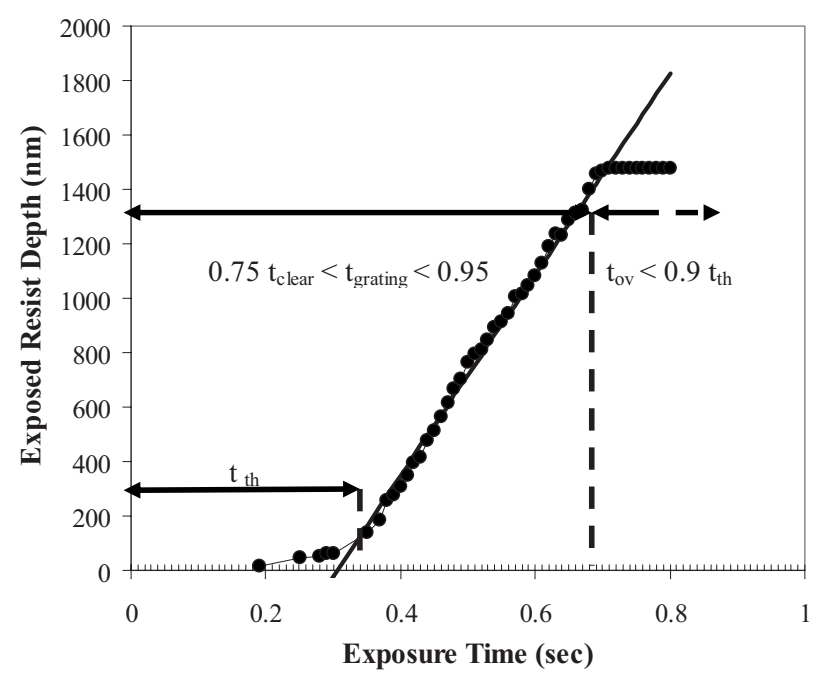

Fig. 1 The response curve for a $1.0-\mu \mathrm{m}$ thick Shipley 1813 photoresist coating is shown. The grating was exposed $\left(t_{\text {grating }}\right)$ by delivering less than $90 \%$ of the saturation dose $\left(t_{\text {clear }}\right)$. The analog intensity function was then overlaid with an exposure dose $\left(t_{\mathrm{ov}}\right)$ value less than the estimated threshold dose $\left(t_{\text {th }}\right)$ such that it will not further expose the resist line areas.

standard photolithographic exposure process. Shipley S1813 photoresist was spin-coated on silicon substrates. Figure 1 shows the measured exposure response curve of the resist. A GCA g-line (436-nm) stepper was used to expose the wafers. The latent image of the grating was formed in the photoresist by initially delivering an exposure dose $\left(t_{\text {grating }}\right)$ that is less than the dose-to-clear value $\left(t_{\text {clear }}\right)$, for the coating at hand, using a conventional chrome-clear amplitude mask. Subsequently, a follow-up overlay exposure $\left(t_{\mathrm{ov}}\right)$, using a phase-only mask and the same stepper tool, induced the desired spatial intensity profile variation. ${ }^{11}$ The value of the second exposure was lower than the threshold dose of the measured resist response curve $\left(t_{\mathrm{ov}}<t_{\mathrm{th}}\right)$. The resist was then developed to obtain gratings with spatially varying duty cycles. The fill fraction at any location within the grating structure was dependent on the local dose delivered from the combined exposure steps. Using the second exposure step to target a window around the optimal exposure dose-to-size for the grating, we were able to preserve the fidelity of the developed photoresist profile while spatially varying the local feature width.

Figure 2 shows the grating profile obtained from the two-step exposure method discussed here, compared to the profile obtained from a standard single grating exposure. The total aerial image at the wafer plane is the sum of the intensity profile of the grating mask [Fig. 2(a)] and the analog intensity profile of the phase mask [Fig. 2(b)]. The combination of the two separates the grating structure in a region with a "nominal" analog bias to the left and a region with a "high" analog bias to the right of the image shown. In the high-intensity region, the grating grooves developed wider than the ones at the nominal exposure region. The standard grating line-space ratio was modulated with proportion to the local cumulative dose variation. By keeping the maximum value of the overlay exposure dose $\left(t_{\mathrm{ov}}\right)$

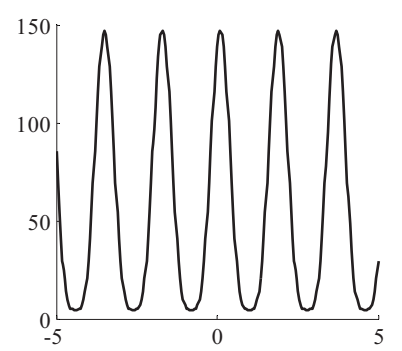

(a)

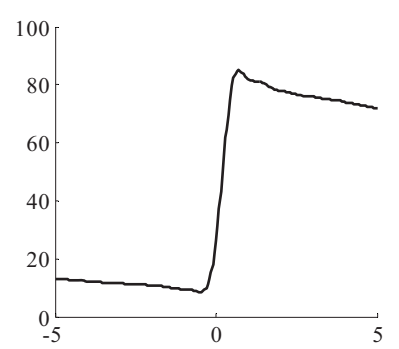

(b)

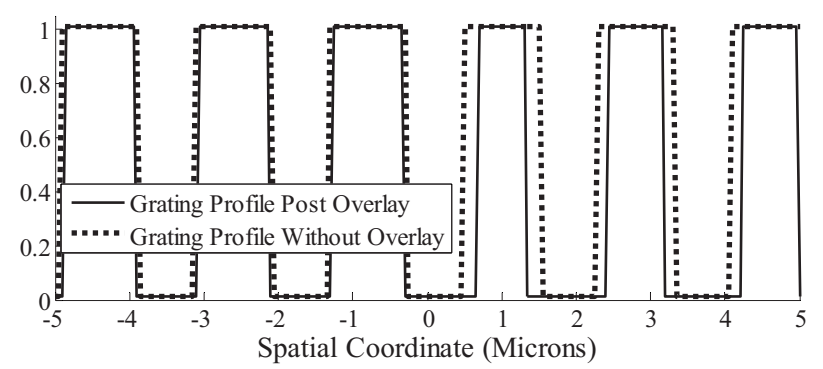

(c)

Fig. 2 (a) The grating aerial image intensity profile due to the first exposure using the grating amplitude mask is shown. (b) The overlaid analog aerial image intensity profile, from the second exposure and (c) the combination of exposures (a) and (b), results in separating the grating structure in a region with a "nominal" analog bias to the left, and a region with a "high" analog bias to the right of the graphic shown. In the "high" intensity region, the grating grooves develop thinner than the ones at the nominal exposure region. For clarity $10 \mu \mathrm{m}$ of the spatial extent is shown in all images.

smaller than the threshold value (Fig. 1), we achieved linewidth modulation without loss in height of the grating structure.

Figure 3 further elaborates on the method used to vary the grating fill fraction. The technique that is discussed here does not significantly modify the shape of the line profile. The linear regime and the range over which the exposed line width can be varied is extended without "pinching" the resist structure to an apex. The local linewidth of the patterned features is a function to the amount of the local dose received from the spatially varying intensity profile of the overlay exposure. This is achieved due to the analog profile of the second exposure dose, which has no line-space features as the grating exposure did initially. At the upper limit, when the second exposure dose is beyond the threshold of the photoresist, the adjacent features overlap, resulting in development of the entire patterned area. Decoupling the grating latent image formation and the line-width modulation steps allows arbitrary fill fraction variation to be achieved. In contrast, for a grating formed with a single exposure, change in exposure dose causes the spatial dose distribution pattern to be modified, resulting in a limited change in line width. If the grating is severely overexposed, in order to force the line width to shrink considerably, the sidewalls will form at an angle smaller than the normal and the grating line will eventually "pinch," forming triangular cross sections.

In order to design structures with a spatial duty-cycle modulation, an accurate model is necessary to predict the 


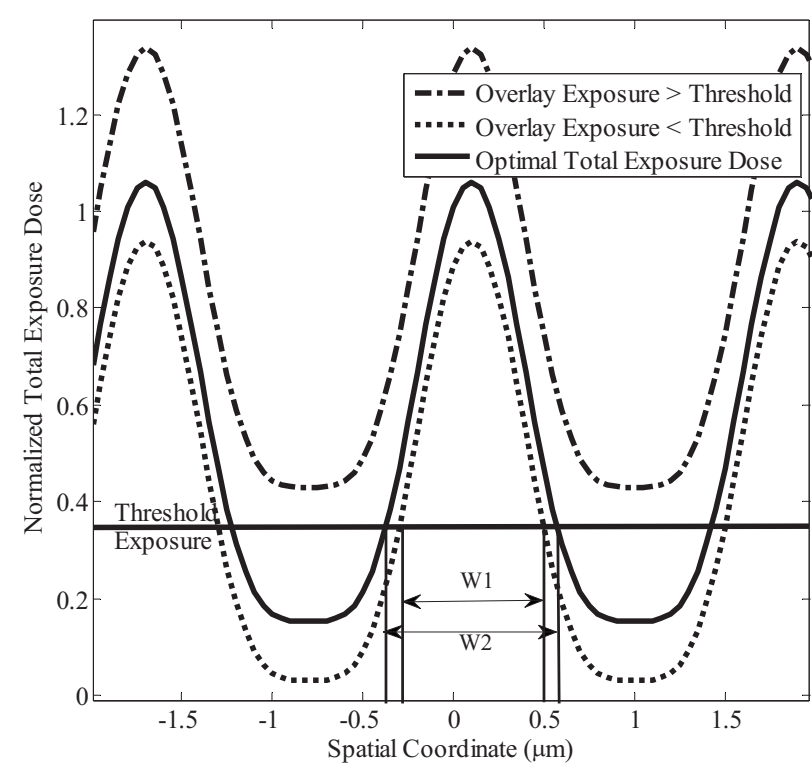

Fig. 3 By using the threshold region to control the combined exposure $\left(t_{\text {grating }}+t_{\mathrm{ov}}\right)$, the line width can be varied over a large range (red lines). In the case that the overlay exposure is greater than estimated threshold $\left(t_{\mathrm{ov}}>t_{\mathrm{th}}\right)$, the patterns are washed out after development because the entire exposure profile is shifted to values above the exposure threshold (blue line).

exposure dose required to achieve a desired local feature width. Results obtained from simulation and experiments for the fabrication of smooth analog variation of line width are presented in the following sections.

\section{Methods}

The variation of grating feature sizes as functions of the exposure dose was studied using a photolithographic process-simulation model. Initially, the exposed aerial image of the stepper was modeled numerically and then the effects of the developer dissolution were calculated. Experimental data were used to extract the matching values for the process parameters. These values were used in the numerical model to accurately predict the variation of the grating fill fraction dependent on the exposure dose profile.

\subsection{Exposure Model}

A mercury arc lamp is used in the stepper and adjusted to provide a Koehler illumination. The spatial frequencies generated by the mask transmittance function at the pupil plane were calculated numerically using a fast Fourier transform computation. Neglecting the partial coherence of the source, this can be represented as ${ }^{12}$

$G(f, g)=H(f, g) F T\left[g_{0}(x, y)\right]$

$g_{i}(x, y)=F T^{-1}[G(f, g)]$.

In Eqs. (1) and (2), $H(f, g)$ represents the transfer function

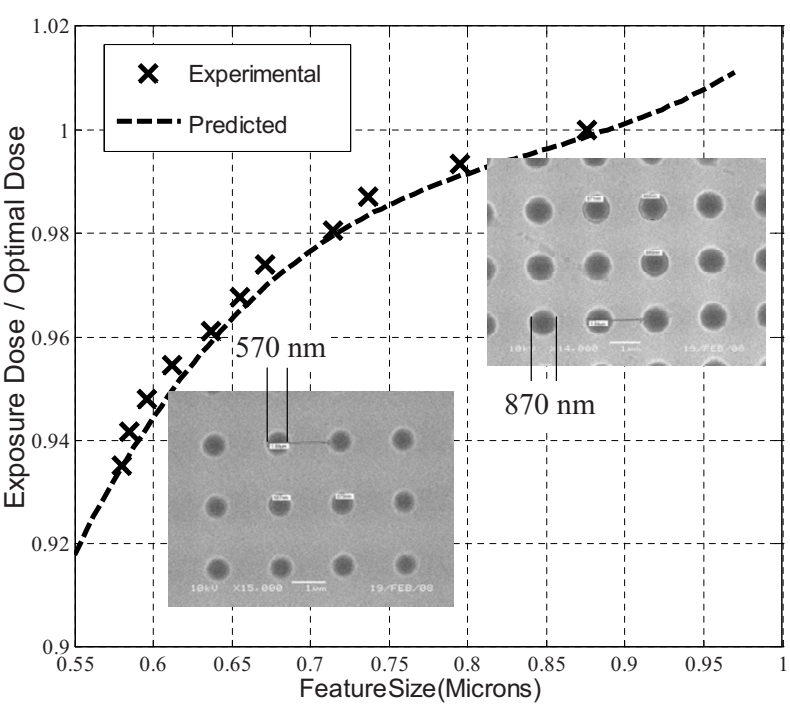

(a)

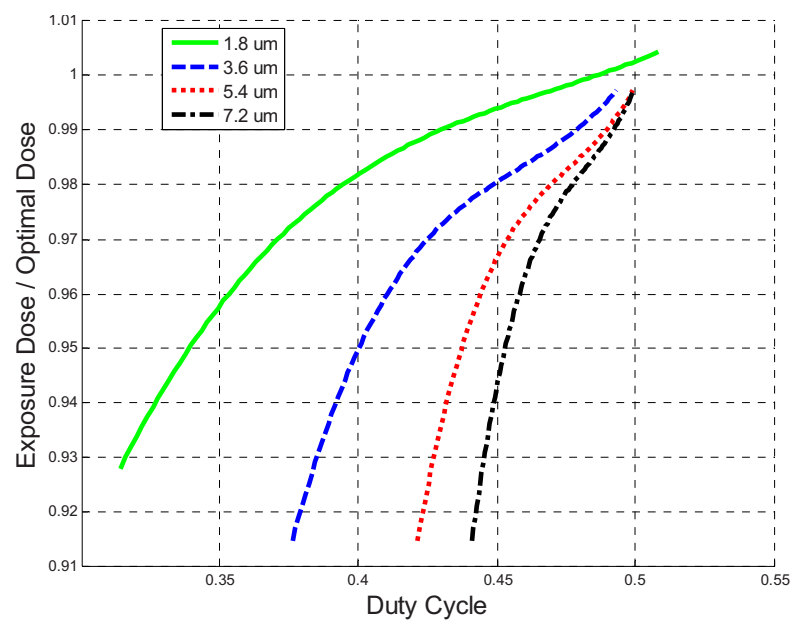

(b)

Fig. 4 (a) Feature size variation as a function of exposure dose: experimental and predicted values for process parameter $\gamma=0.6$ and (b) predicted dose variation as a function of the grating period, based on the same simulation model.

of the stepper, and $G(f, g)$ is the Fourier spectrum of the object amplitude $g_{0}(x, y)$ at the pupil plane.

The partial coherence was accounted for by multiplying the object amplitude function with the plane-wave component of the spatial-frequency source point. The angular extent of the source is represented by the partial coherence factor. For the GCA 6300 stepper used in this work, it was taken to be $0.6{ }^{13}$ The image intensity in the resist was computed by incorporating the depth, as a defocus effect, and the standing wave pattern within the photoresist layer. ${ }^{14}$ The photoactive compound concentration (PAC) was calculated from the Dill parameters. ${ }^{15}$ The effect of the second exposure is computed by updating the PAC concentration from the result of the initial exposure value. The photoresist development rate function was computed from the PAC using the four-parameter Mack model, ${ }^{16}$ and the final photoresist profile was obtained.

The method outlined above leads to deviations from the 


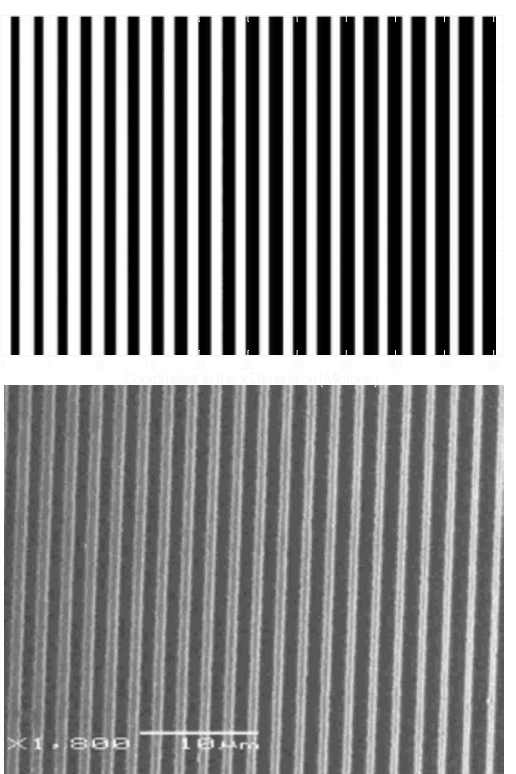

(a)

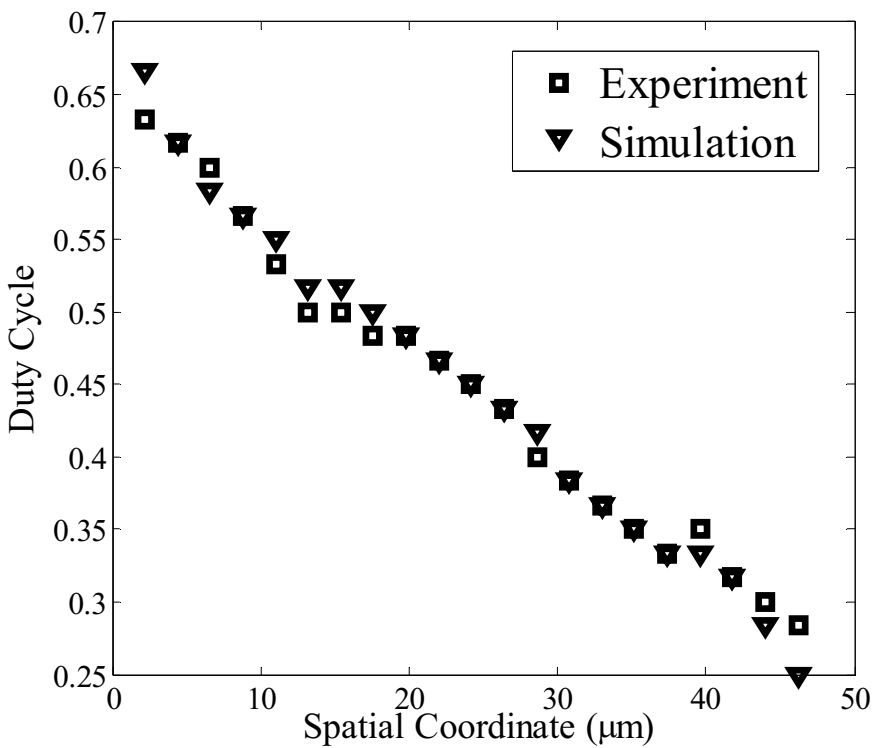

(b)

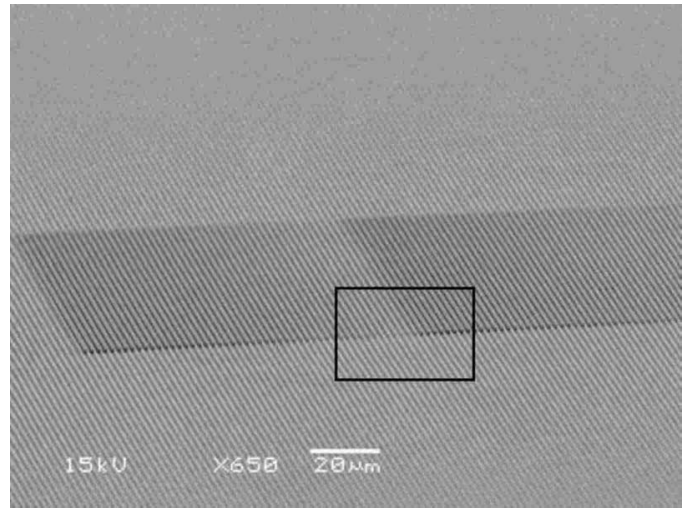

(c)

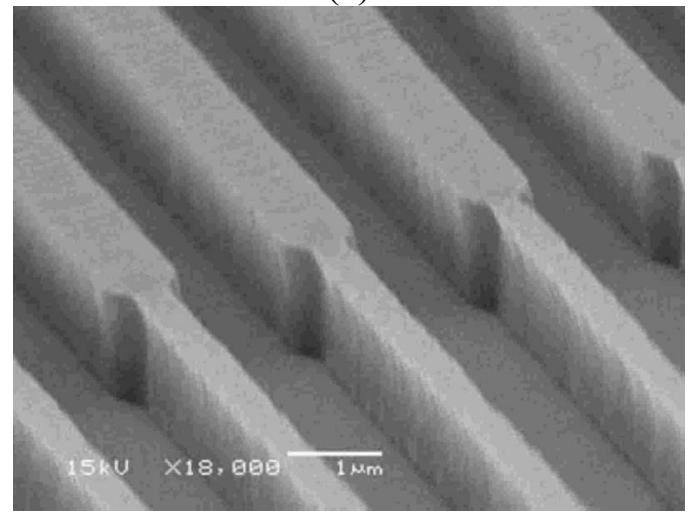

(e)

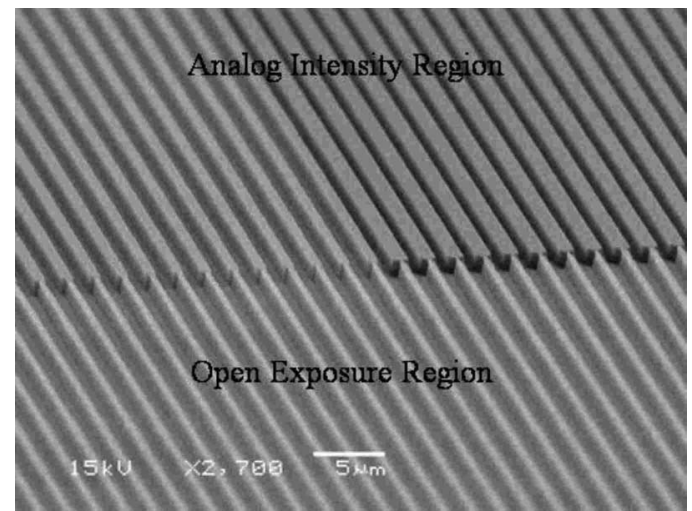

(d)

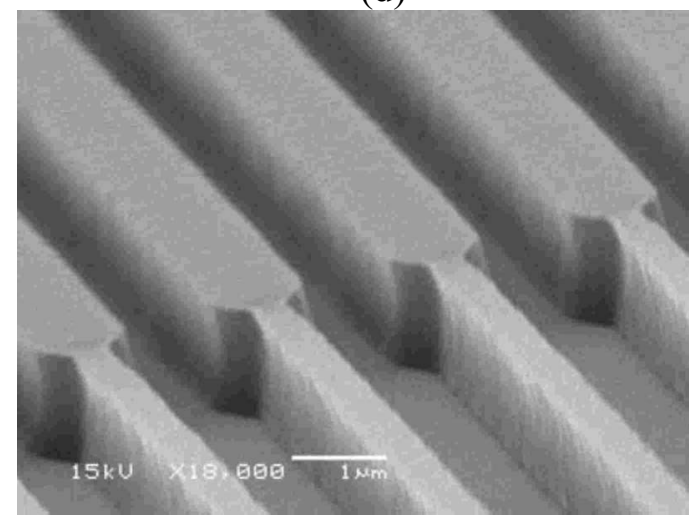

(f)

Fig. 5 (a, b) Comparison of numerical and experimental results for the duty-cycle variation obtained with a 1-D grating exposure overlaid with an analog intensity function, (c, d) SEM images of the variable fill fraction gratings; and (e, f) magnified images of the transition from the phase mask controlled analog intensity region to the open aperture in the low and high fill-fraction regions.

actual line widths obtained by experiment. ${ }^{17}$ One of the reasons for such deviations is that a real photoresist absorbs the incident radiation, resulting in a decreased image contrast and normalized image log slope. ${ }^{18}$ We were able to obtain an accurate prediction of the line-width variation with the dose by applying adjusted values for the optimal process parameters extracted from experimental data.

The exposure effect in a high-contrast photoresist can be expressed as ${ }^{18}$ 


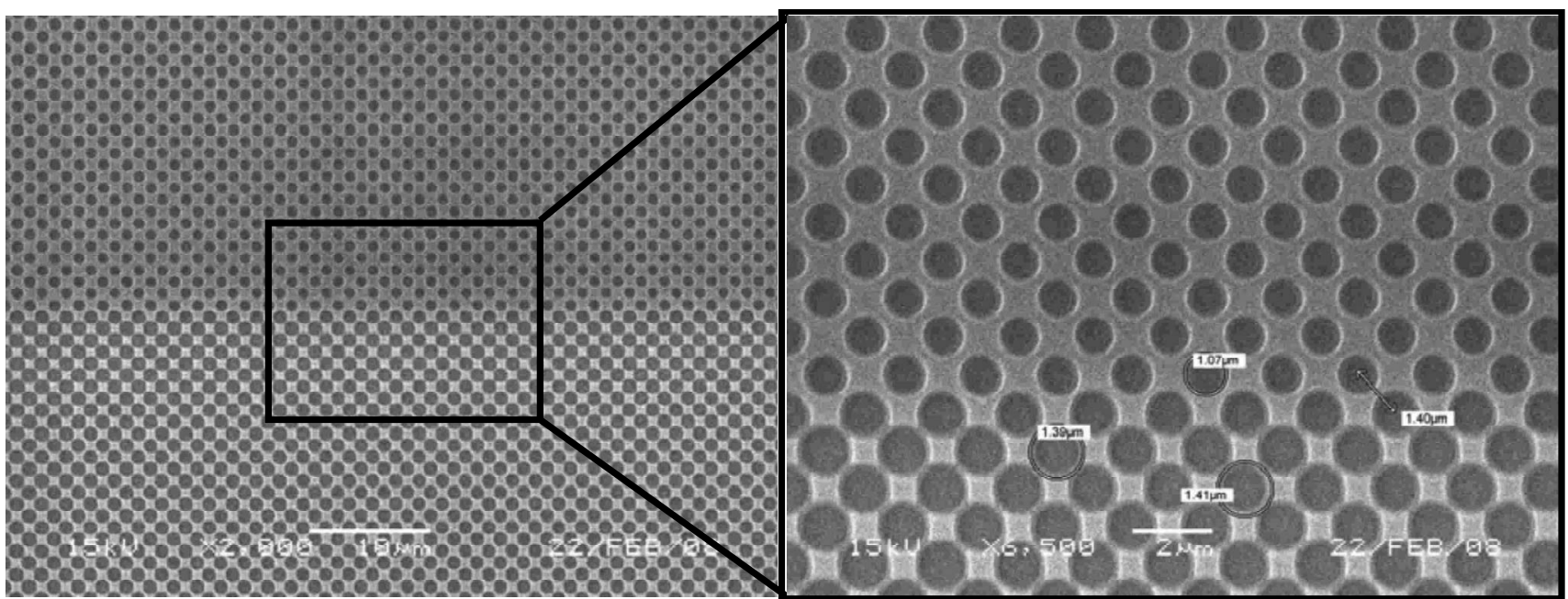

(a)

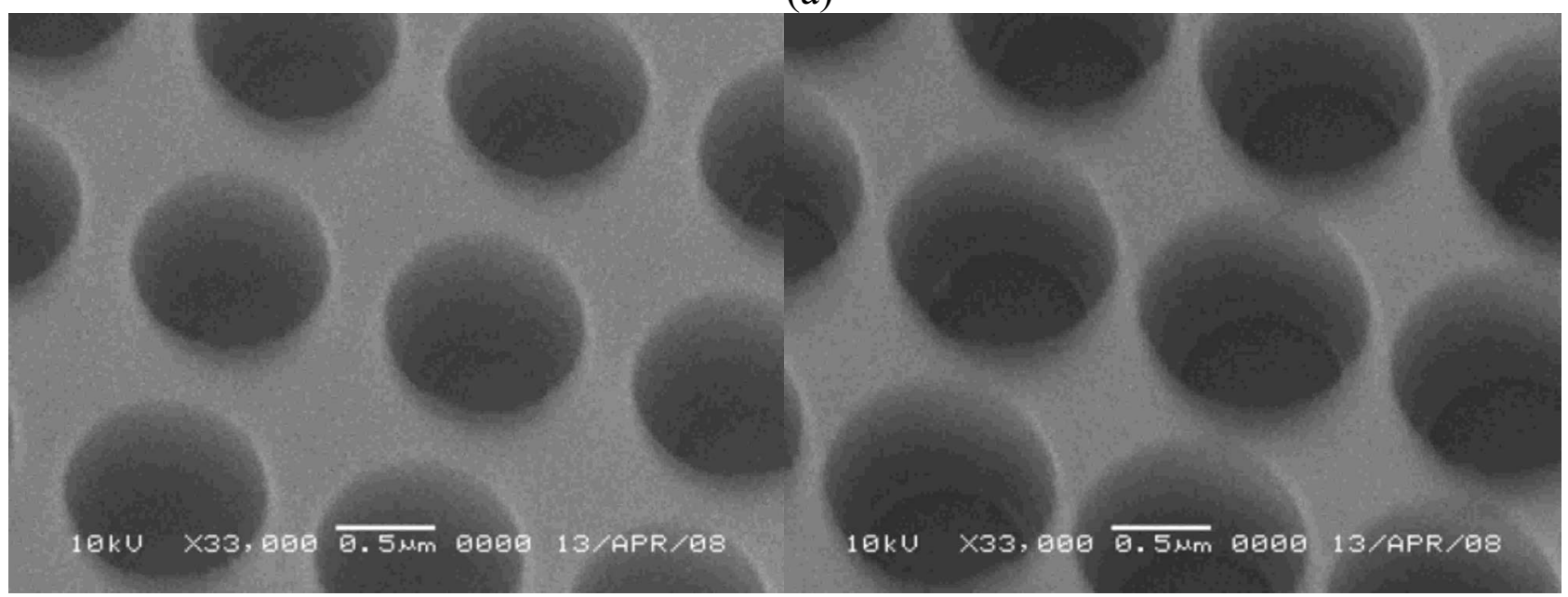

(b)

Fig. 6 The effect of an analog exposure ramp profile overlaid on a 2-D grating. (a) As the overlay dose increases, the adjacent holes overlap and the structure transitions from a hole array to a post array. (b) Magnified SEM images of the photoresist, taken at a 30 deg tilt, showing the sidewall and cleared photoresist at different spatial coordinates in the patterns shown in (a). The profile of the structures is slightly conical, with a larger opening on the air side. This is attributed to resist loss during development.

$E_{x}=E_{N}\left(\frac{1}{I_{x}}\right)^{\gamma}$

where $E_{N}$ is the energy required for the photoresist to be cleared for exposure through a mask less open aperture, $E_{x}$ is the optimum energy of exposure for a given dose to size, $I_{x}$ is the normalized image intensity for a given pattern, and $\gamma$ is a process parameter that relates the change in development rate function for unit change in exposure energy. For a high-contrast photoresist, $\gamma$ assumes a value very close to unity. The change in feature size for a change in exposure energy can be used to calculate $\gamma$ experimentally. Using this, we can obtain the experimental value of $\gamma$ for the photoresist process from the duty cycle to dose curve. In order to convert the simulated aerial image intensity pattern to the resist threshold energy, we used a modified equation,
$E_{w}=E_{x}\left(\frac{I(w / 2)}{I(0)}\right)^{\gamma}$

where $I(w / 2)$ is the threshold intensity required for resist exposure using the given pattern obtained from the resist model, and $I(0)$ is the intensity at the center of the feature obtained from the simulated aerial image intensity. These values are used to predict the required exposure $\left(E_{w}\right)$ for the desired feature width $(w)$.

\subsection{Fabrication}

Silicon substrates were coated with a $1-\mu$ m-thick layer of Shipley 1813 photoresist. One-dimensional (1D) and twodimensional (2D) gratings of $2-\mu \mathrm{m}$ periods were partially exposed in the photoresist, using an amplitude grating chrome mask, by delivering a dose between 75 and $90 \%$ of the saturation dose estimated for the pattern, through a 

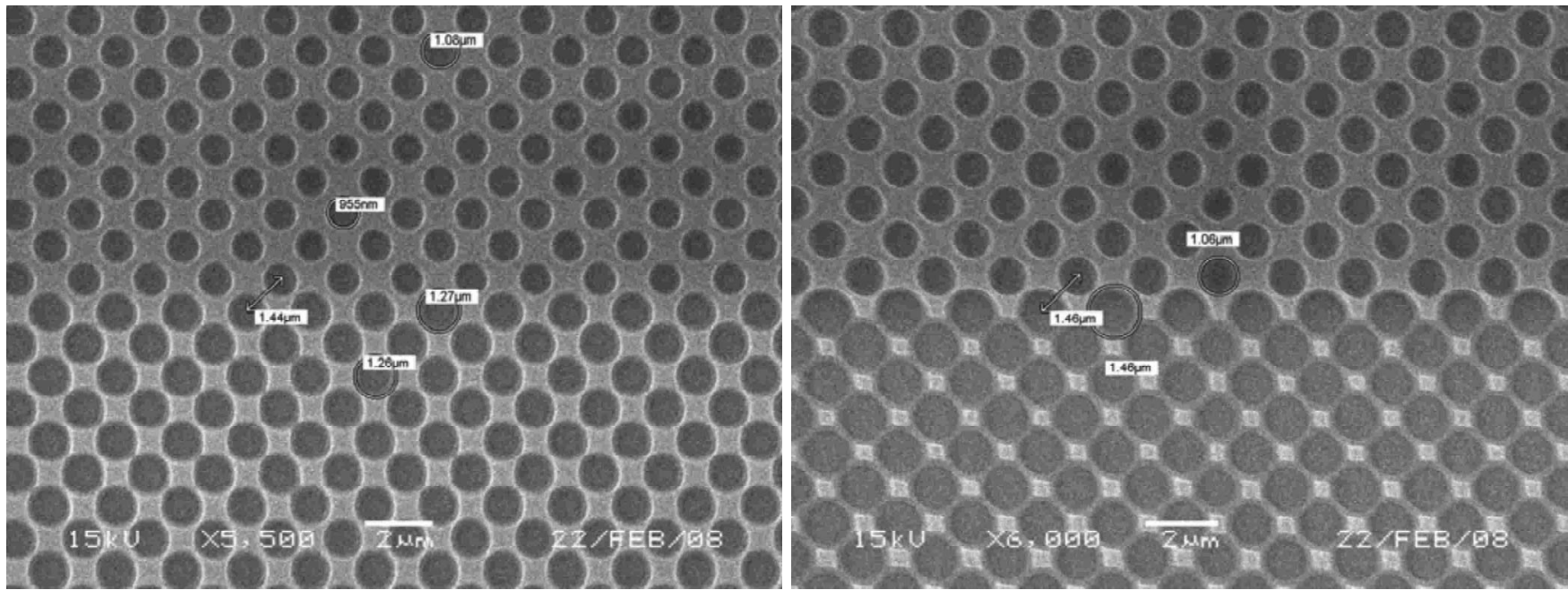

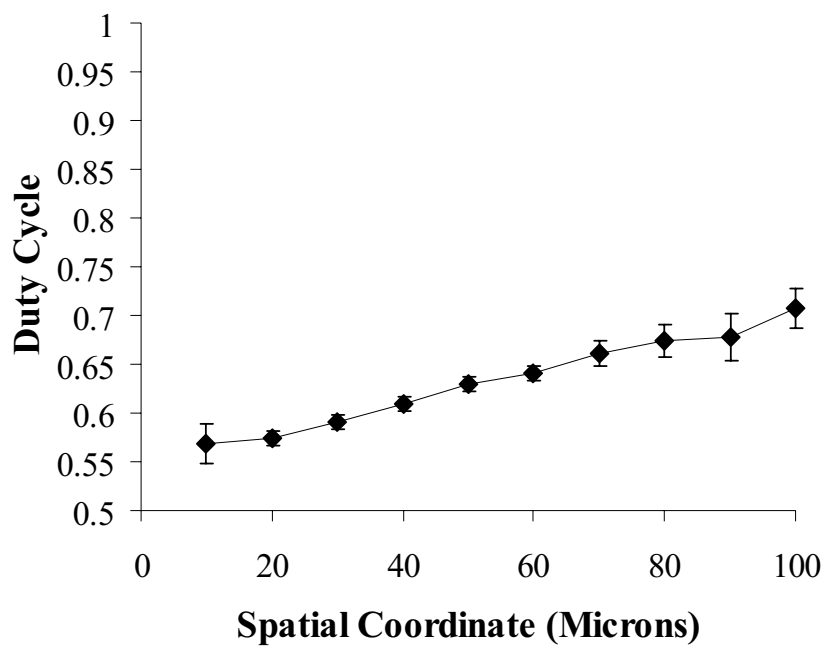

(a)

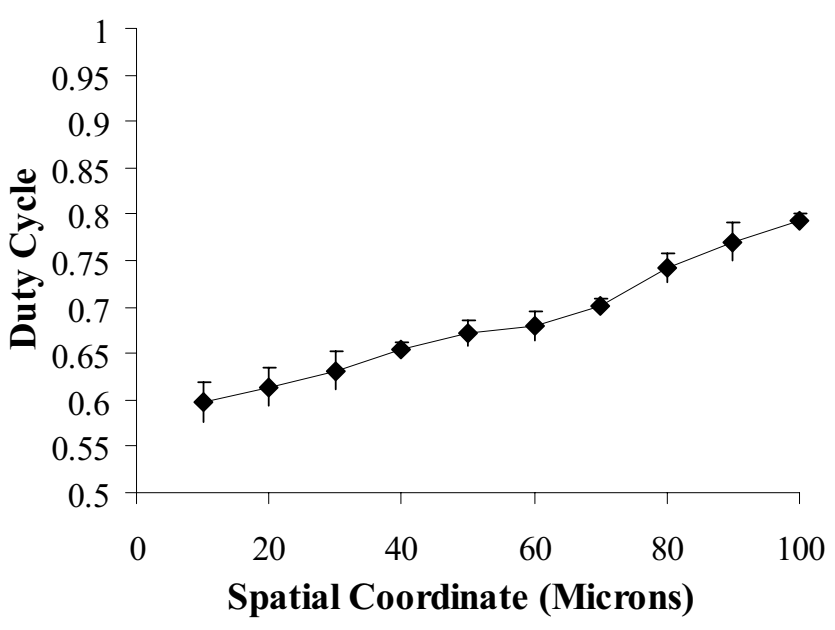

(b)

Fig. 7 Quantitative results from the exposures of the 2-D grating of Fig. 6. The duty-cycle variation range was adjusted by varying the analog exposure dose $\left(t_{\mathrm{ov}}\right)$. The resulting fill fraction of the grating varied from (a) $0.53-0.7$ and from (b) 0.6-0.8 in the SEM images.

GCA g-line (436-nm) stepper. This dose was designed to be lower than the dose-to-clear value $\left(t_{\text {clear }}\right)$, by an amount of energy smaller than the absolute value of the dose to threshold $\left(t_{\text {clear }}-t_{\text {grating }}<\left|t_{\text {th }}\right|=0.3 \mathrm{~s}\right)$ shown in Fig. 1. Following this, an analog intensity profile generated using a phase-only mask function was overlaid. Details of the mask used to generate the analog intensity profile are found elsewhere. ${ }^{11}$ The second exposure dose was chosen to satisfy two important criteria. First, the highest intensity in the overlaid exposure profile was maintained less than the threshold exposure of photoresist $\left(t_{\mathrm{ov}}<\left|t_{\mathrm{th}}\right|=0.3 \mathrm{~s}\right)$. This was to ensure that the photoresist left unexposed by the first exposure was not removed upon development, to preserve the fidelity of the formed grating without variation in line height across the device. In addition, enough energy was delivered so that the photoresist in the grating grooves formed by the initial partial exposure was saturated at the point of the minimum exposure intensity required to satisfy the dose-to-size value. The range of duty-cycle modulation was controlled by: $(i)$ the exposure dose of the grating, (ii) the differential intensity of the designed phase grating transmission, ${ }^{11}$ and (iii) by the exposure dose delivered during the overlay exposure.

In order to quantitatively compare the modulation effect on the duty cycle to the overlay exposure dose, the experiment was repeated using the grating mask and a clear field mask to deliver the same values of $t_{\text {grating }}$ and $t_{\mathrm{ov}}$. The second exposure dose $\left(t_{\mathrm{ov}}\right)$ was kept below the threshold value of the photoresist, as in the case of the phase mask trial. The modulation of the duty cycle with the cumulative exposure dose was measured using a scanning electron microscope (SEM). The measured values were compared to the results of the lithographic model.

\section{Results and Discussion}

The correlation between experimental and predicted exposure dose required to form a desired feature width is shown in Fig. 4. The curves were generated using a 50\% duty- 
cycle mask. When $\gamma=0.6$, as estimated from the experimental data, the predicted values of the exposure dose-tosize correlate within $3 \%$ of the measured values. The range of modulation in the duty cycle with exposure dose is smaller as the grating period increases. At the long period limit, large variations in exposure dose are required to significantly change the duty cycle. The variation in feature size with exposure dose is however independent of the grating period because the feature-size modulation is dependent on the partial coherence factor, the numerical aperture of the imaging system, and the process parameters of the photoresist. Essentially, the described technique allows the photoresist response to the absorbed radiation and subsequent development to change from a depth variation to a width variation of the exposed features. By operating in a process window around the dose to clear and using an analog spatial intensity distribution, we were able to achieve uniform variation in grating duty cycle.

For the case of subwavelength grating structures, the dependence of the slope of the feature-width variation with exposure dose to the grating period allows one to change the range and position of the duty-cycle variation for a constant spatial intensity profile. This corresponds directly to a change in refractive index and the phase depth obtained across the fabricated optical element. The same intensity profile can also be overlaid on 1-D gratings of different orientation to form polarization-sensitive apodized gratings.

A comparison of the simulated and experimental results for a one-dimensional grating overlaid with an analog exposure is shown in Fig. 5. The measured spatial modulation of the duty cycle is in close agreement with our model, with a linear dependence across the element, in accordance with the functional form of the analog intensity overlay. SEM images of the variable fill fraction gratings in the low and high fill-factor areas are shown in Figs. 5(c)-5(f). The abrupt change in fill fraction of the gratings along the grooves is due to the transition in the phase-only mask from an area of analog intensity, to an open aperture that passes all of the incident radiation.

Spatially varying structures were also formed using 2-D gratings exposed in the photoresist, followed by the analog intensity exposure. Experimentally, a phase mask with ana$\log$ intensity profiles that formed index prisms was used. Uniform, analog variation of duty cycles was obtained. SEM images of refractive prisms formed using spatially variant grating structures is shown in Fig. 6. As the fill fraction of the 2-D grating holes increases across the element, adjacent holes merge, and the structure transitions into a "post" array. As observed with the linear grating, the photoresist height of the 2-D grating does not change across the device. As the dose increases from the bottom to the top in Fig. 6, the adjacent holes overlap and the structure transitions from an array of holes to an array of posts. Magnified micrographs of the photoresist taken at a $30 \mathrm{deg}$ tilt show the sidewall profile and cleared photoresist height at different locations in the device. The profile of the structures is slightly conical, with a larger opening on the air side. This is attributed to resist loss during development.

By varying the level of the grating exposure $\left(t_{\text {grating }}\right)$ and the analog profile function $\left(t_{\mathrm{ov}}\right)$, we were able to change the range of duty-cycle variation. The effect of analog exposure time was studied by holding the initial grating exposure dose at $85 \%$ of the saturation dose value and by delivering different doses of the prism intensity. For the same analog intensity profile, we were able to position and control the duty-cycle variation range as shown in Fig. 7. The duty cycle was varied between 0.5 and 0.7 , and from 0.6 to 0.8 , for different overlay exposures. This corresponds to an ability to engineer the effective refractive index range of the fabricated subwavelength optical functions. The grating exposure dose delivered can be used to control the central placement of the duty-cycle modulation. Because the dutycycle variation with exposure dose has different linear and nonlinear regimes, this effect can be used to realize complex optical functions.

\section{Conclusions}

We have demonstrated a simple, low-cost, high-fidelity process for the fabrication of complex optical structures. We have presented a method for the simulation of feature size variations with exposure dose, using an empirical modeling method. The effects of the various process parameters were used to refine the numerical model, and the predicted results closely matched our experiment. The ability to fabricate large-dimension subwavelength variable-modulation gratings has been demonstrated, leading to their use in practical applications. The fabrication methodology developed in this paper allows control over the placement, range, and limits of duty-cycle variation in 1-D and 2-D grating structures, enabling more challenging designs to be realized easily and cost effectively. Combining this method with various approaches to generate analog intensity overlay profiles enables the design and implementation of artificialindex optical function devices.

\section{Acknowledgment}

This work was funded in part by the National Science Foundation CAREER Grant No. ECS 0348280.

\section{References}

1. M.-S. L. Lee, P. Lalanne, and P. Chavel, "Blazed-binary diffractive elements with periods much larger than the wavelength," J. Opt. Soc. Am. A 17(7), 1250-1255 (2000).

2. C. Sauvan, P. Lalanne, and M.-S. L. Lee, "Broadband blazing with artificial dielectrics," Opt. Lett. 29(14), 1593-1595 (2004).

3. Z. E. Bomzon, G. Biener, V. Kleiner, and E. Hasman, "Radially and azimuthally polarized beams generated by space-variant dielectric subwavelength gratings," Opt. Lett. 27(5), 285-287 (2002).

4. W. Mohammed, M. Pitchumani, A. Mehta, and E. G. Johnson, "Selective excitation of the $\mathrm{LP}_{11}$ mode in step index fiber using a phase mask," Opt. Eng. 45(7), 074602 (2006).

5. A. Mehta, J. D. Brown, P. Srinivasan, R. C. Rumpf, and E. G. Johnson, "Spatially polarizing autocloned elements," Opt. Lett. 32(13), 1935-1937 (2007).

6. D. Wiesmann, C. David, R. Germann, D. Erni, and G. L. Bona, "Apodized surface-corrugated gratings with varying duty cycles," IEEE Photonics Technol. Lett. 12(6), 639-641 (2000).

7. D. Wiesmann, R. Germann, G.-L. Bona, C. David, D. Erni, and H Jacket, "Add-drop filter based on apodized surface-corrugated gratings," J. Opt. Soc. Am. B 20(3), 417-423 (2003).

8. G. Biener, A. Niv, V. Kleiner, and E. Hasman, "Geometrical phase image encryption obtained with space-variant subwavelength gratings," Opt. Lett. 30(10), 1096-1098 (2005).

9. L. L. Mane-Si, S. Bansropun, O. Huet, S. Cassette, B. Loiseaux, A. Wood, C. Sauvan, and P. Lalanne, "Sub-wavelength structures for broadband diffractive optics," Proc. SPIE 6029, 602919 (2006).

10. A. Mehta, R. C. Rumpf, Z. Roth, and E. G. Johnson, "Nanofabrication of a space-variant optical transmission filter," Opt. Lett. 31(19), 2903-2905 (2006)

11. J. Sung, H. Hockel, and E. G. Johnson, "Analog micro-optics fabrication by use of a two-dimensional binary phase-grating mask," Opt Lett. 30(2), 150-152 (2005). 
12. A. K. Wong, Resolution Enhancement Techniques in Optical Lithography, SPIE Press, Bellingham, WA (2001).

13. J. Sung, M. Pitchumani, and E. G. Johnson, "Aberration measurement of photolithographic lenses by use of hybrid diffractive photomasks." Appl. Opt. 42(11), 1987-1995 (2003).

14. C. A. Mack, "Analytical expression for the standing wave intensity in photoresist," Appl. Opt. 25(12), 1958 (1986).

15. F. H. Dill, W. P. Hornberger, P. S. Hauge, and J. M. Shaw, "Characterization of positive photoresist," IEEE Trans. Electron Devices 22(7), 445-452 (1975)

16. C. A. Mack, "Absorption and exposure in positive photoresist," Appl. Opt. 27(23), 4913 (1988).

17. T. A. Brunner and R. A. Ferguson, "Approximate models for resist processing effects," Proc. SPIE 2726, 198-207 (1996)

18. R. Hershel and C. A. Mack, Lumped Parameter Model for Optical Lithography, Academic Press, New York (1987)

Pradeep Srinivasan is a $\mathrm{PhD}$ candidate at the College of Optics and Photonics/CREOL/FPCE at the University of Central Florida. He earned a master's degree in electrical engineering from the University of Cincinnati, Ohio, in 2003. His current research interests include design and fabrication of space-variant 3-D micro- and nanooptical elements. He is a student member of SPIE.

Zachary A. Roth is a PhD student at the University of North Carolina, Charlotte. He received his bachelor of science in optical engineering from the Rose-Hulman Institute of Technology in 2004. He completed his master of science in optics at CREOL, University of Central Florida, in 2006. He is currently a graduate research assistant for Dr. Eric Johnson at the University of North Carolina, Charlotte, where his research interests include 3-D micro-optics.

Menelaos K. Poutous is a research scientist with the Center for Optoelectronics and Optical Communications of the Charlotte Research Institute, University of North Carolina, Charlotte. Prior to this position he was with Digital Optics Corporation as a senior and then principal development engineer from the year 2000 to 2007 . He held the position of teaching faculty at Emory University in Atlanta, Georgia, from 1993 to the year 2000. He received his doctorate in physics from the School of Physics of the Georgia Institute of Technology in 1996. His research interests are in diffractive micro- and nanooptical elements, photolithographic fabrication processes applied to wafer-scale micro-optics, and applications of diffractive elements in laser cavities and devices. He is a member of the Optical Society of America and SPIE.
Eric G. Johnson is a program director in the electronics, photonics, and device technologies program in the Electrical, Communications and Cyber Systems (ECCS) Division of the National Science Foundation (NSF). He serves the NSF as an IPA and holds a joint appointment as a professor of physics and optical science, as well as electrical and computer engineering, at the University of North Carolina, Charlotte. He also serves as the associate director of the Center for Optoelectronics and Optical Communications. He was previously an associate professor at the College of Optics and Photonics, Center for Research and Education in Optics and Lasers (CREOL) at the University of Central Florida, where he now holds a courtesy appointment. Prior to joining CREOL, he was the vice president of research and development at Digital Optics Corporation. His research spans the area of micro-optics and nanophotonics, with particular emphasis on active and passive photonic devices. He has over 100 publications in the field with 9 issued patents. Dr. Johnson currently serves on the board of directors for SPIE. He was also the previous chair for the Optics in Information Science Division of the Optical Society of America (OSA) and the former OSA technical group chair for holography and diffractive optics. He is currently a topical editor for Applied Optics and a previous associate editor for $\mathrm{JM}^{3}$. He is a fellow of SPIE and a member of OSA and IEEE. He received his $\mathrm{PhD}$ in electrical engineering from the University of Alabama at Huntsville, his MS in electrical sciences from the University of Central Florida, and a BS in physics from Purdue University. 